\title{
Dietary Patterns and Associations with Chronic Diseases of Ageing: Evidence from The Irish Longitudinal Study on Ageing (TILDA)
}

\author{
$\underline{\text { Deirdre O'Connor }}^{1}$, Siobhan Scarlett ${ }^{2}$ and Rose Anne Kenny ${ }^{1}$ \\ ${ }^{1}$ Trinity College Dublin, Dublin 2, Ireland and \\ ${ }^{2}$ Trinity College Dublin, Dublin, Ireland
}

\section{Abstract}

In the field of nutritional epidemiology, principal component analysis (PCA) has been used to derive dietary patterns, but this has never been conducted in a large, nationally representative sample of older adults in Ireland. The aim was to identify dietary patterns amongst older adults in Ireland derived through PCA and to examine cross-sectional associations with common comorbidities of ageing.

PCA was performed using data from Wave 3 of TILDA (2014), a nationally representative cohort of community-dwelling adults aged $\geq 54(n=4,395)$. We derived major dietary patterns from a PCA of reported intake from a Food Frequency Questionnaire (FFQ). Objective measures for Body Mass Index (BMI), diabetes (HbAlc), bone density(heel bone ultrasound), blood pressure and total cholesterol were collected during a health assessment. The Mini-Mental State Examination (MMSE) was also performed during health assessment with trained interviewers, as a measure of global cognitive function.

Statistical analyses were conducted using multivariate logistic regression, adjusting for age, sex, marital status, income, educational attainment, alcohol consumption, smoking and physical activity.

With the use of PCA, five dietary patterns were identified in the sample - Pattern 1 (fresh fruit, vegetable, fresh fish and dairy), Pattern 2 (confectionary, fatty and processed foods), Pattern 3 (meats, processed meats and salty foods), Pattern 4 (carbohydrates and processed foods) and Pattern 5 (savoury snacks, spreads and processed foods). Those characterized by Pattern 4 were more likely to be overweight (adjusted RRR $0.12,95 \%$ CI $0.05-0.20 ; p=0.001$ ), obesity (adjusted RRR $0.18,95 \%$ CI $0.10-0.27 ; p=0.001$ ) and have diabetes (adjusted RRR $0.10,95 \%$ CI $0.01-0.19 ; \mathrm{p}=0.024$ ) after adjusting for known covariates.

Our results suggest that in the older adult population of Ireland, a dietary pattern typified by consumption of refined grains and processed carbohydrates is associated with higher prevalence of overweight, obesity and diabetes.

\section{Conflict of Interest}

There is no conflict of interest 